\title{
Lunar Dust and Lunar Simulant Activation and Monitoring
}

\section{W.T. Wallace ${ }^{1}$, D.K. Hammond ${ }^{2}$, and A.S. Jeevarajan ${ }^{3}$}

${ }^{1}$ USRA/NASA Johnson Space Center, ${ }^{2}$ EASI/NASA Johnson Space Center, and ${ }^{3}$ NASA Johnson Space Center

\section{Introduction}

Prior to returning to the moon, understanding the effects of lunar dust on both human physiology and mechanical equipment is a pressing concern, as problems related to lunar dust during the Apollo missions have been wel documented (J.R. Gaier, The Effects of Lunar Dust on EVA Systems During the Apollo Missions. 2005, NASA-Glenn Research Center. p. 65). While efforts were made to remove the dust before reentering the lunar module, via brushing of the suits or vacuuming, a significant amount of dust was returned to the spacecraft, causing various problems. For instance, astronaut Harrison Schmitt complained of "hay fever" effects caused by the dust, and the abrasive nature of the material was found to cause problems with various joints and seals of the spacecraft and suits. It is clear that, in order to avoid potential health and performance problems while on the lunar surface, the reactive properties of lunar dust must be quenched.

It is likely that soil on the lunar surface is in an "activated" form, i.e. capable of producing oxygen-based radicals in a humidified air environment, due to constant exposure to meteorite impacts, UV radiation, and elements of the solar wind. An activated silica surface serves as a good example. An oxygen-based radical species arises from the breaking of Si-O$\mathrm{Si}$ bonds. This system is comparable to that expected for the lunar dust system due to the large amounts of agglutinic glass and silicate vapor deposits present in lunar soil. Unfortunately, exposure to the Earth's atmosphere has passivated the active species on lunar dust, leading to efforts to reactivate the dust in order to understand the true effects that will be experienced by astronauts and equipment on the moon.

Electron spin resonance (ESR) spectroscopy is commonly used for the study of radical species, and has been used previously to study silicon- and oxygen-based radicals, as well as the hydroxyl radicals produced by these species in makes them unattractive for the monitoring of lunar dust activity. A more suitable technique is based on the change in luorescence of a molecule upon reaction with a hydroxyl radical (or other radical species). Fluorescence instruments ar much less costly and bulky than ESR spectrometers, and small fluorescence sensors for space misstons have already been developed (F. Gao, et al., J. Biomed. Opt. 10 (2005) 054005).

For the current fluorescence studies, the terephthalate molecule has been chosen for monitoring the production of hydroxyl radicals in solution. As shown in Scheme 1, the reaction between the non-fluorescent terephthalate molecule and a hydroxyl radical produces the highly-fluorescent 2-hydroxyterephthalate molecule. This reaction has been shown to

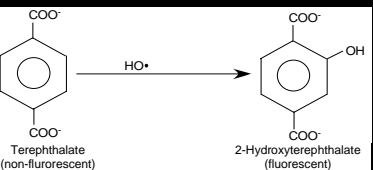

Scheme 1: Reaction of hydroxyl radicals with non-
fluoressent terephthalate to produce fluorescent 2.
hydroxyterephthalate.

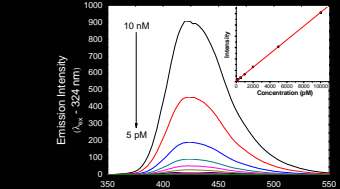

Figure 1: Fluorescence of

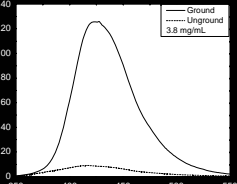

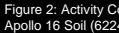

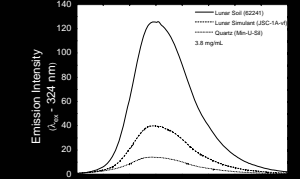

Wavelength (nm)
Figure 3: Activity Comparison of Freshly Ground Apollo 16 Soil,

\section{Results}

of a small amount of unactivated materials to a $10 \mathrm{mM}$ terephthalate solution produces very little fluorescence (not shown). However, it can be seen that the general order or hydroxyl radical production decreases in the order lunar dust > lunar simulant $>$ quartz. Grinding of the materials was shown to have a large effect. For instance, grinding of Apollo 16 soil (62241) produces the spectrum in Figure 2. With the spectrum produced by the unground material also shown, and a comparison to the calibration curve, it is easy to see that simply grinding the lunar dust produces a large amount of hydroxyl radicals. The amount of radicals is even more striking when the lunar dust, lunar simulant, and quartz are compared. In Figure 3, it can be seen that, under the same grinding conditions, lunar soil produces 2-3 times more hydroxyl radicals than lunar simulant and 10 times more than quartz.

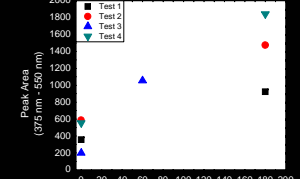

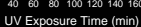

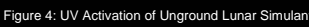

NHBE with Lunar Simulant

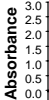
2.5 (1)

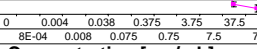
Concentration $[\mathrm{mg} / \mathrm{mL}]$

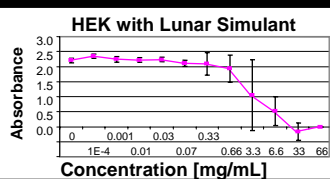

Figure 6: Effect of Unactivated Lunar Simulant on Skin
Cells. (courtesy M.J. Cunningham)
Figure 5: Effect of Unactivated Lunar Simulant on Lun
Cells. (courtesy M.J. Cunningham)
Ideally, due to the fact that grinding changes the particle size distribution of the sample, other methods would be used to reactivate the samples. One possibility is to mimic the ultraviolet radiation experienced by the lunar soil. Initial experiments have been carried an 800 W UV lamp. As can be seen in Figure 4, after performing the terephthalate fluorescence test, it can be seen that exposure to UV radiation does indeed lead to an increase in reactivity. initial cellular viability studies have been performed. Figures 5 and 6 show the changes in viability of human lung (NHBE) and skin (HEK) cells when exposed to varying concentrations of unground lunar simulant. For each of cell strains, very large concentrations of dust were required to see a change in viability. However, cell death is no the only possible effect of exposure to lunar dust. It is possible that exposure to these materials could cause the cells to produce othe harmful species, such as hydrogen peroxide, or to damage the cell 作 death at lower concentrations. Further studies are needed in order to determine the full effects of lunar dust on cells.

\section{Conclusions}

This work has demonstrated methods of activating quartz, lunar simulant, and lunar soil, and monitoring the levels of activation using fluorescence assay based on the production of hydroxyl radicals in solution. Ground quartz, a known carcinogen, was shown to be much less active for the production of hydroxyl radicals than ground lunar simulant and ground lunar soil. Additionally, the same assay has shown that exposing unground lunar simulant to a high-powered ultraviolet source (to mimic the UV radiation obtained by soil on the lunar surface) can also lead to activation. Concurrent studies on the toxicity of lun ar sim high doses of unground simulant are required in order to cause cell death. Each of these results provide evidence of the need for full studies on these materials prior to returning to the lunar surface. out by exposing unground lunar simulant under moderate vacuum to

As the effect of lunar dust on the human body is of major interest

- Fluorescence testing procedure: Ground and unground material were added to $15 \mathrm{~mL}$ centrifuge tubes containing 2.5 $\mathrm{mL}$ of $10 \mathrm{mM}$ terephthalate dissolved in PBS. The mixtures were allowed to interact for 30 minutes before being filtered using $0.22 \mu \mathrm{m}$ syringe filters. Two $\mathrm{mL}$ of the filtered solution was added to a quartz fluorescence cuvette, and the fluorescene spectrum was obtained using a Perkin-Elmer LS $50 \mathrm{~B}$ spectrometer.

- Calibration procedure: The spectra and calibration curve shown in Figure 1 were produced using 2-hydroxyterephthalate - Callbration procedure. The spectra and callibration curve shown in Figure 1 were produced using 2-hydroxyterephthala material was then purified using recrystallization until the fluorescence spectra were maximized. Mass spectrometry also confirmed that the only species present following recrystallization was 2-hydroxyterephthalate. A $1 \mathrm{mM}$ solution was confirmed that the only species present following recrystallization was 2-hydroxyterephthalate. A $1 \mathrm{mM}$ solution was above $10 \mathrm{nM}$, the fluorescence saturated the detector. Dilutions below this threshold varied linearly with concentration, as above $10 \mathrm{nM}$, the fluorescence saturated the detector. Dilution
shown in the figure. The excitation wavelength was $324 \mathrm{~nm}$.

\section{Future work}

Future work will focus on a variety of tests involving lunar dust activation, deactivation, dissolution properties, and cellular toxicity.

- Proton beam and further UV exposure experiments w - The deactivation kinetics of activated lunar dust

the development of dust mitigation programs.

- Dissolution studies vill be carred out to deternine if any potentilly harmful species are leached into solution and to determine their concentrations.

- Further cellular toxicity experiments will be conducted using different cell lines in order to determine the effects of lunar dust activation in vitro and to determine if different cells are more susceptible to damage from lunar dust.

\section{Acknowledgements}

Lunar Airborne Dust Toxicity Advisory Group (LADTAG), D.H. Garrison, R.K. Bradley, Ph.D., M.J. Cunningham, Ph.D. Prof. J. Wise 